\title{
Structure of Microtubule-Based Microtentacles
}

\author{
Alison N. Killilea ${ }^{1}$, Roseann Csencsits ${ }^{1}$, Sam Kenny ${ }^{1,2}$, Ke Xu ${ }^{1,2}$, Kenneth H. Downing ${ }^{1}$
}

${ }^{1}$ Life Science Division, Lawrence Berkeley National Laboratory, Berkeley, CA
${ }^{2}$ Department of Chemistry, University of California Berkeley, Berkeley, CA

Microtentacles (McTNs) are thin projections found on circulating tumor cells (CTCs) that can be tens of micrometers long. They have been recognized as a potentially critical factor in metastasis due to their involvement in attachment of CTCs to secondary sites of metastasis. So far little structural characterization has been reported, other than that these projections are based on microtubules (MTs), unlike most other cell projections that are based on actin. Work published to date strongly suggests that stabilization of McTNs increases the risk of metastasis through enhanced reattachment of CTCs [1-3]. Stabilization occurs by a number of factors that often increase during progression of cancer, including increased expression of proteins such as vimentin and tau [2, 4]. Of particular clinical importance, Taxol stabilizes MTs and as a result stabilizes McTNs. Since Taxol is widely used in treatment of cancer, it is very important to understand the magnitude and structural basis for any increased risk of metastasis. In addition, developing a structural understanding for these effects would help to identify novel drug targets that could compensate for any added risk that treatment with drugs such as Taxol poses. With the goals of understanding McTN structure and the mechanisms by which stabilization of McTNs promote reattachment, we have used cryo-electron microscopy and tomography, scanning electron microscopy (SEM) and stochastic optical reconstruction microscopy (STORM) to characterize the structure and activity of these protrusions.

SEM was used to follow the development of McTNs after drug treatment of MDA-MB-231 breast cancer cells with latrunculin (LA) and Taxol, with results shown in Fig. 1. A time course series showed formation of McTN within 3 minutes of drug addition (data not shown), and at 15 minutes (Figure 1b) McTNs longer than $10 \mu \mathrm{m}$ can be seen. We have studied their molecular composition by fluorescence labeling. New advances in super-resolution microscopy have allowed for lateral resolution of $10 \mathrm{~nm}$ and axial resolution of $20 \mathrm{~nm}$, which is ideal for investigating the arrangement of $10 \mathrm{~nm}$ intermediate filaments (IFs) and 25nm MTs inside McTNs. Using dual-objective STORM we imaged MTs and vimentin IFs in suspended MDA-MB-231 breast cancer cells treated with LA and Taxol. In general, the filaments inside the McTNs are arranged parallel to each other but appear spatially separated (Fig. 2).

Resin embedding and sectioning were used to provide a view of structures within thicker parts of the McTNs near the cell body. MDA-MB-231 cells treated with LA and Taxol show structural details including the IFs, MTs and ribosomes (Fig. 3). The separation between MTs and IFs inside the McTN suggests that another protein may be linking these networks. Immunofluorescence experiments revealed the presence of plectin, a cytoskeletal linker protein, inside McTNs (data not shown).

Cryo tomography has been used to obtain clear 3D maps of the MT distribution within thin parts of the McTNs (Fig. 4). So far we have seen no IFs in these regions, supporting the idea that these two components of the cytoskeleton are not tightly interconnected, even though there is evidence that vimentin helps to stabilize McTNs [4]. 

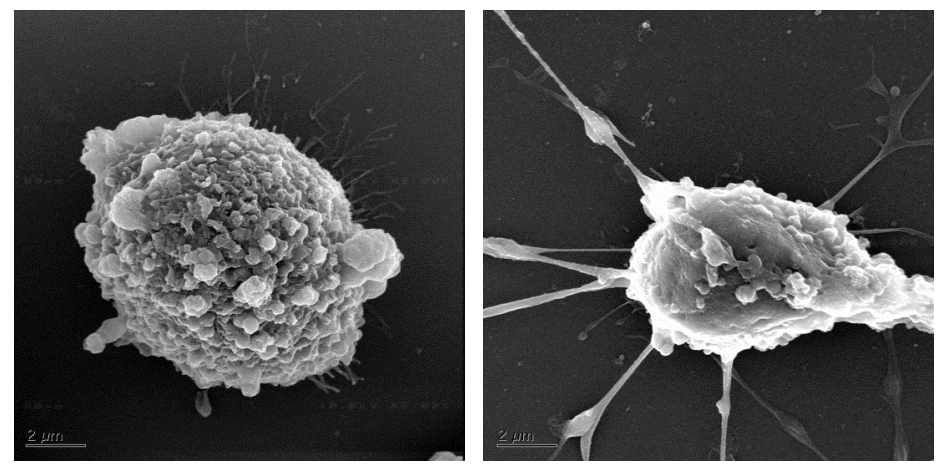

Figure 1. McTN visualized by SEM. MDA-MB231 cells were suspended over ultra low attachment plates, treated with $5 \mu \mathrm{M} \mathrm{LA}$ and $1.7 \mu \mathrm{M}$ Taxol for 15 minutes. Cells were fixed in $2.5 \%$ glutaraldehyde followed by ethanol dehydration, critical point drying and gold-palladium sputter coating. Left: control; right: drug treated cell.

Figure 3. TEM visualization of internal McTN structure. Microtubules (MT) and intermediate filaments (IF) are visible, although they are often found in different regions of the sample. MDA-MB-231 cells were suspended over ultra low attachment plates and treated with $5 \mu \mathrm{M}$ LA and 1.7 $\mu \mathrm{M}$ Taxol for 30 minutes before application to polylysine treated, carbon-coated sapphire discs followed by fixation in $2 \%$ paraformaldehyde and $0.5 \%$ glutaraldehyde. Discs were high pressure frozen, freeze substituted and resin embedded.

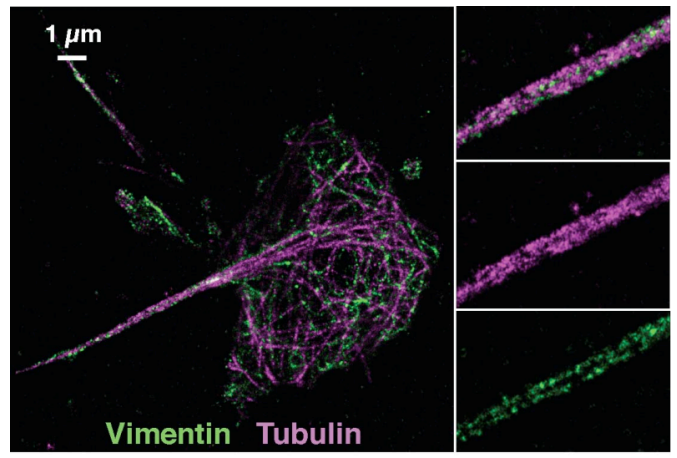

Figure 2. STORM imaging of MT and IF networks in McTN. Glutaraldehyde-fixed cells were labeled with mouse DM1 $\alpha$ antitubulin and chicken polyclonal anti-vimentin antibodies, washed, and AlexaFluor 647 and CF680 secondaries were applied.

Figure 4. Cryo-electron tomography of McTN. (top) Section $\sim 3 \mathrm{~nm}$ thick through 3D tomographic reconstruction obtained from tilt series of low-dose images of frozen-hydrated cell. Parts of several microtubules can be seen passing up and down through this section. (bottom) Model for distribution of MTs enclosed by membrane of McTN derived from full 3D map.
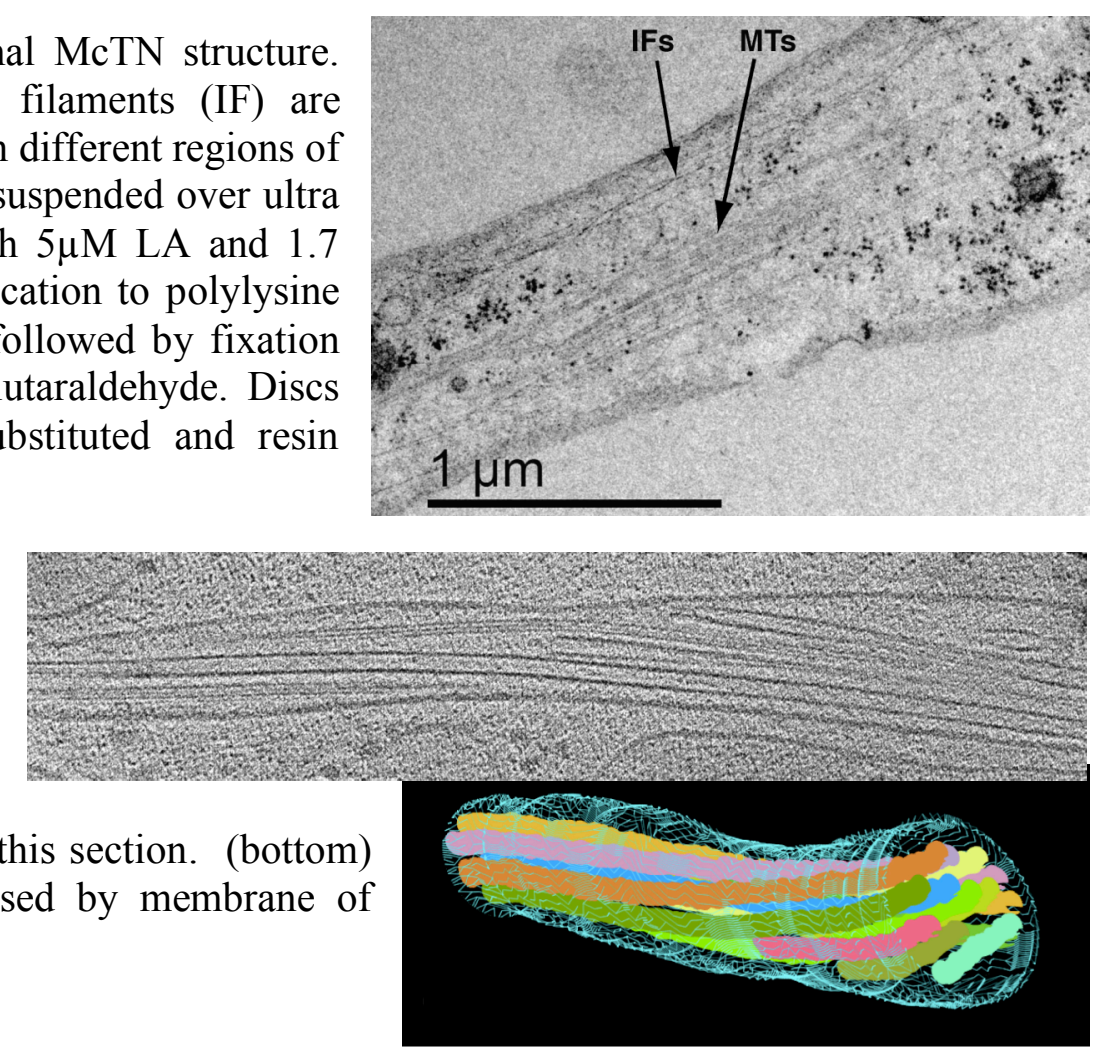

References:

[1] Balzer EM, et al, Oncogene 29 (2010), 6402.

[2] Matrone et al, Oncogene 29 (2010), 3217.

[3] Charpentier MS, et al, Cancer Res. 74 (2014), 1250.

[4] Whipple RA, et al, Cancer Res. 68 (2008), 5678. 